\title{
SOLUBILIZATION OF HEN BRAIN NEUROTOXIC ESTERASE IN DIMETHYLUULOXIDE
}

\author{
B.W. Schwab, C-S.G. Davis, P.H. Miller, and R.J. Richardson \\ Toxicology Program, School of Public Health, \\ The University of Michigan, Ann Arbor, Michigan 48109
}

Received August 15, 1985

Neurotoxic esterase is the putative site of initiation of organophosphorusinduced neuropathy. The protein is membrane-associated and will thus require solubilization before it can be purified. Its enzymic activity was retained in hen brain microsomes suspended in $10-60 \%(\mathrm{v} / \mathrm{v}$ in water) dimethylsulfoxide and 5-20\% dimethylacetamide, but lost in 5-20\% 1- and 2-propanol as well as higher concentrations of dimethylsulfoxide. Soluble activity $(100,000 \times \mathrm{g}$, $60 \mathrm{~min}$ supernatant) was not obtained with dimethylacetamide, but $24 \%$ of the recovered activity $(67 \%)$ was solubilized in $40 \%$ dimethylsulfoxide, with retention of its native response to inhibitors. Solvent extraction of active enzyme is of intrinsic interest and expands the options for its purification. (c) 1985 Acaderic Press, Inc.

NTE is a protein found in highest concentration in brain and lymphocytes. It possesses hydrolytic activity in vitro for several lipophilic esters, and on the basis of its inhibition by ops, the enzyme is presumed to be a serine esterase. Its activity is characterized by relative resistance to paraoxon $\left(\mathrm{pI}_{50}\right.$ 3.5) and sensitivity to mipafox $(\mathrm{pI} 505.1)\left(20 \mathrm{~min}\right.$ incubation at $\left.37^{\circ} \mathrm{C}\right)$. Evidence which has been extensively reviewed $(1,2)$ suggests that phosphorylation of NTE by neuropathic OPs is the initial step in the molecular pathogenesis of a neuropathy occurring in humans and other animals exposed to these compounds. Non-neuropathic inhibitors of NTE (a small number of phosphinates, sulfonates, and carbamates) protect against neuropathy from subsequent ly administered neuropathic OPs, presumably by preventing phosphorylation of the active site by neuropathic OPs $(3,4)$. Thus, mere inhibition of enzyme activity is ruled out as the mechanism of initiation of neuropathy. It has been noted that only neuropathic ops are capable of undergoing aging (cleavage of a side chain from the phosphoryl moiety

Abbreviations: NTE, neurotoxic esterase; $O P(s)$, organophosphorus compound(s); Paraoxon, diethy1 4-nitrophenylphosphate; Mipafox, N,N'-di-2-propy1 phosphorodiamidofluoridate; DMSO, dimethylsulfoxide; DMA, dimethylacetamide; IRE, inhibitor-resistant esterase; EDTA, ethylenediaminetetraacetic acid, disodium salt. 
subsequent to phosphorylation of the esterase), implicating this reaction as the second necessary step in the mechanism of neurotoxicity (4-7).

Bulk purification and molecular characterization of NTE would aid in determining how its phosphorylation and aging initiate neuropathy. Because NTE is membrane-associated ( 8 ), it must be solubilized before undertaking purification. Mild treatments such as homogenization in media of high or low ionic strength are insufficient to solubilize NTE (2). Initial attempts with detergents yielded a solubilized species (non-sedimentable at $100,000 \mathrm{x} \mathbf{g}$, $60 \mathrm{~min}$ ) that was resistant to paraoxon, but with significantly greater sensitivity to mipafox than exhibited by the native enzyme (9). Subsequent work with lower detergent concentrations has yielded soluble NTE preparations with retention of native inhibitor responsiveness (10-12). It was of interest to examine organic solvent systems for the solubilization of NTE to expand options for purification, to facilitate enzymological characterization with water-insoluble substrates, and to obviate detergent removal in membrane reconstitution experiments (13). This report details the relative stability and extractability of hen brain microsomal NTE in aqueous solutions of DMSO, DMA, 1-propanol and 2-propano1. Due to the association of IRE activity with NTE in hen brain microsomal membranes (8), data on its solubilization are included for comparison in the more detailed studies with DMSO.

\section{MATERLALS AND METHODS}

Chemicals: DMSO, DMA, 1-propanol, and 2-propanol were obtained from Burdick and Jackson Laboratories (Muskegon, MI), Sigma Chemical Co. (St. Louis, MO), Eastman Kodak (Rochester, NY), and MCB Manufacuting Chemists (Cincinnati, $O H$ ), respectively. The solvents were mixed with water in the concentrations $(\% \mathrm{v} / \mathrm{v})$ noted in Figure 1 . Reagents for esterase assays were purchased from Sigma, except 4-aminoantipyrine (Eastman Kodak). Mipafox and phenyl valerate were synthesized in this laboratory, according to methods described by Johnson (12). All other chemicals were analytical reagent grade or the highest grade commercially available; deionized-distilled water was used for all aqueous solutions. Buffer $\mathrm{pH}$ values are corrected to $25^{\circ} \mathrm{C}$.

Tissue Preparation: Adult laying hens (Omega Chicks, Haslett, MI) were killed by decapitation and brains removed and rinsed with ice-cold water. A $10 \%(w / v)$ homogenate of brain in water was made with 10 strokes of a glassteflon homogenizer (clearance, $0.25 \mathrm{~mm}$ ) fitted to a $2300 \mathrm{RPM}$ drill press. The homogenate was transferred to a polypropylene tube and centrifuged for $20 \mathrm{~min}$ at $10,000 \times \mathrm{g}$. This and all subsequent centrifugations were carried out at $4^{\circ} \mathrm{C}$. An aliquot of the supernatant was pipetted into a polycarborate 
centrifuge bottle and centrifuged at $100,000 \times \mathbf{g}$ for $60 \mathrm{~min}$. The resulting (microsomal) pellet was used for all studies.

Solubilization: Microsomal pellets were resuspended at $0-4^{\circ} \mathrm{C}$ in solvent mixtures (a volume equivalent to the original volume of supernatant) with the aid of a hand-held glass homogenizer ( 10 strokes). The suspensions were assayed for NTE activity. In solvent systems where there was adequate preservation of NTE activity (arbitrarily set at $75 \%$ of the specific activity of a control suspension of microsomes in water), the suspension was placed in a clean polycarbonate bottle and again centrifuged at $100,000 \mathrm{x} g$ for $60 \mathrm{~min}$. Supernatants and pellets (resuspended in the same solvent system) were then assayed for NTE, IRE, and protein. Aliquots of some supernatants were centrifuged at $100,000 \times \mathbf{g}$ for an additional $60 \mathrm{~min}$. Recovery (\%) of enzyme activity or protein content $=100 \times$ (supernatant + pellet activity or content)/ (original suspension activity or content). Solublization $(\%)=100$ $x$ (supernatant activity or amount)/(original suspension activity or amount).

Esterase Assay: Phenyl valerate hydrolys is by NTE and IRE was assayed using the method of Johnson (14) with the following modifications. A11 volumes were halved from the original method and preincubation was done in buffer (50 mM Tris/0.20 mM EDTA, pH 8.0) containing $100 \mathrm{uM}$ paraoxon, or $100 \mathrm{uM}$ paraoxon plus 50 uM mipafox. Phenyl valerate concentration was adjusted upward to $0.50 \mathrm{mg} / \mathrm{ml}$ incubate and the reaction was allowed to continue for 30 min. Aliquots of suspensions and supernatants were diluted with the appropriate solvent mixture or buffer to provide 20-40 ug protein per assay tube in a volume of $500 \mathrm{ul}$ while the pellet suspensions were adjusted to 10-20 ug protein/ $500 \mathrm{ul}$. NTE-dependent phenol production was determined from the absorbance difference between samples preincubated with paraoxon or paraoxon plus mipafox. IRE activity was determined from the absorbance in samples preincubated with paraoxon plus mipafox, less the absorbance in a substrate blank. Mipafox titration curves of NTE activity were generated by preincubating samples in buffer containing 100 uM paraoxon plus $0-200$ uM mipafox for $20 \mathrm{~min}$ at $37^{\circ} \mathrm{C}$ and assaying for residual NTE activity.

Protein assay: Protein was assayed by the method of Bradford (15) using the Biorad protein assay reagent (Bio-Rad Laboratories, Richmond, CA). Bovine serum albumin, fraction V (Sigma Chemical Co.) was used for reference standards and made to contain the equivalent concentration of solvents in the unknowns being assayed.

\section{RESULTS}

The effect of solvents on NTE activity in microsomal suspensions is shown in Figure 1. Suspensions of microsomes in aqueous DMSO, 10-60\% (v/v) and DMA, $5-20 \%$ had NTE specific activities in excess of $75 \%$ of that found in a control microsomal suspension in water and were centrifuged to investigate the solubilizing capacity of the solvents. DMSO, $80 \%$ as well as 1 - and 2propanol mixtures (5-20\%) produced substantial loss of activity and were not studied further. High-speed supernatants from aqueous DMSO suspensions contained NTE activity in excess of that observed in supernatants from water suspensions and increased with increasing DMSO concentration. Polycarbonate centrifuge bott1es were visibly damaged by $60 \%$ DMSO; therefore, data obtained 

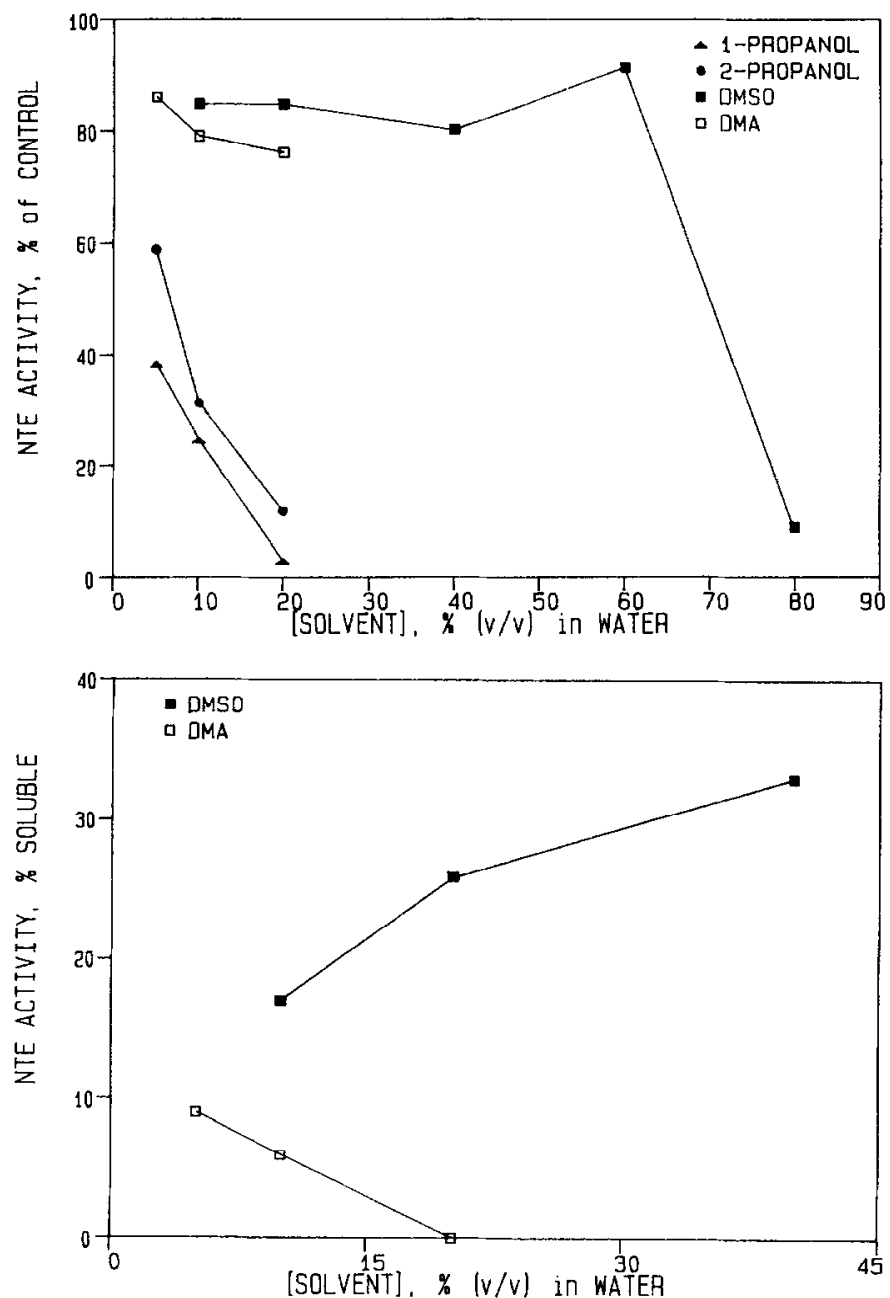

Figure 1. TOP PANEL: Retention of NTE activity in hen brain microsomal membranes suspended in increasing concentrations $(\% \mathrm{v} / \mathrm{v})$ of $1-$ and 2 -propanol, DMSO, and DMA in water; BOTTOM PANEL: NTE activity (\% of recovered activity) in the soluble phase after centrifugation of microsomal suspensions in increasing concentrations ( $\% \mathrm{v} / \mathrm{v}$ ) of DMSO or DMA in water at $100,000 \mathrm{xg}$ for $60 \mathrm{~min}$ at $4^{\circ}$ C. Retention of NTE activity in water was defined to be $100 \%$; NTE activity solubilized in water was $9 \%$. Recoveries following centrifugation were $73-79 \%$ of the original homogenate activities. Data are duplicate determinations from a representative experiment.

using this concentration were considered suspect. However, concentrations of 10,20 , and $40 \%$ DMSO were compatible with polycarbonate and solubilized 17 , 26 , and $33 \%$ of the recovered NTE activity, respectively (recoveries were $73-$ $79 \%$ of the activity in the original suspensions). Solubilization in $5 \%$ DMA was equivalent to that seen with water alone, and was progressively worse in 10 and $20 \%$ DMA. 
Table 1. Solubilization of Hen Brain Microsomal Membranes*

\begin{tabular}{|c|c|c|c|c|}
\hline & \multicolumn{2}{|c|}{ Z Recovery } & \multicolumn{2}{|c|}{ \% Soluble } \\
\hline & Water & $40 \%$ DMSO & Water & $40 \%$ DMSO \\
\hline NTE Activity & $73 \pm 7(6)$ & $67+3(10)$ & $\underline{6+2(6)}$ & $24+3(14)$ \\
\hline IRE Activity & $64 \pm 12(5)$ & $67 \pm 8 \quad(9)$ & $10 \pm 3(5)$ & $35+4(14)$ \\
\hline Protein & $94+11$ & $90+4 \quad(9)$ & $27 \pm 7$ (4) & $51+3(14)$ \\
\hline
\end{tabular}

* Membranes were homogenized in water or $40 \%$ (v/v) DMSO in water and centrifuged at $100,000 \mathrm{xg}$ for $60 \mathrm{~min}$ to give a soluble fraction and pellet. Z Recovery $=100 \times$ (soluble + pellet activity or concentration)/(origina 1 microsomal suspension activity or concentration); \% Soluble = \% recovered activity or concentration in soluble fraction. NTE activity in original microsomal supension in $40 \%$ DMSO was $99+8 \%$ (8) of the activity in water. Values are reported as meantSEM ( $n)$. There are no statistical differences between solvents in recovery values; all values for soluble fractions in $40 \%$ DMSO are statistically larger than those for water ( $p<0.05$, unpaired t-test); soluble IRE activity in $40 \%$ DMSO is statistically larger than soluble NTE activity in $40 \%$ DMSO ( $p<0.05$, unpaired $t$-test).

Based on its overall superiority to the other solvent systems investigated, $40 \%$ DMSO was selected for further evaluation. Recovery and solubilization values for NTE, IRE, and protein in these studies are compiled in Table 1. Suspension of microsomes in $40 \%$ DMSO caused no loss of activity, but high-speed centrifugation resulted in a loss of NTE and IRE recoveries of about $30 \%$ both in water and $40 \%$ DMSO, with little or no loss in protein recoveries. Significantly more NTE, IRE, and protein was solubilized in DMSO than in water. Mipafox titration curves of NTE activity in the microsomal suspension and in the soluble fraction from $40 \%$ DMSO were nearly congruent, with identical $\mathrm{pI}_{50}$ values of 4.72 (Figure 2). Centrifugation of DMSOsolubilized material at $100,000 \times \mathrm{g}$ for an additional $60 \mathrm{~min}$ diminished NTE and IRE activity in the supernatant, although no pellet was detected. Specific activities of NTF and IRE in starting supernatants were $39.6 \pm 7.6(S E M, n=6)$ and $39.3+6.3(n=5) \mathrm{nmol} / \mathrm{min} / \mathrm{mg}$, respectively, but fell to $23.2 \pm 4.8(\mathrm{n}=6)$ and $24.3 \pm 5.7 \mathrm{nmol} / \mathrm{min} / \mathrm{mg}(\mathrm{n}=6)$, respectively, after recentrifugation. These decreases are statistically significant (paired t-test, $p<0.05$ ). In contrast, the differences found in protein concentrations in original $(276+38 \mathrm{ug} / \mathrm{ml})$ and 


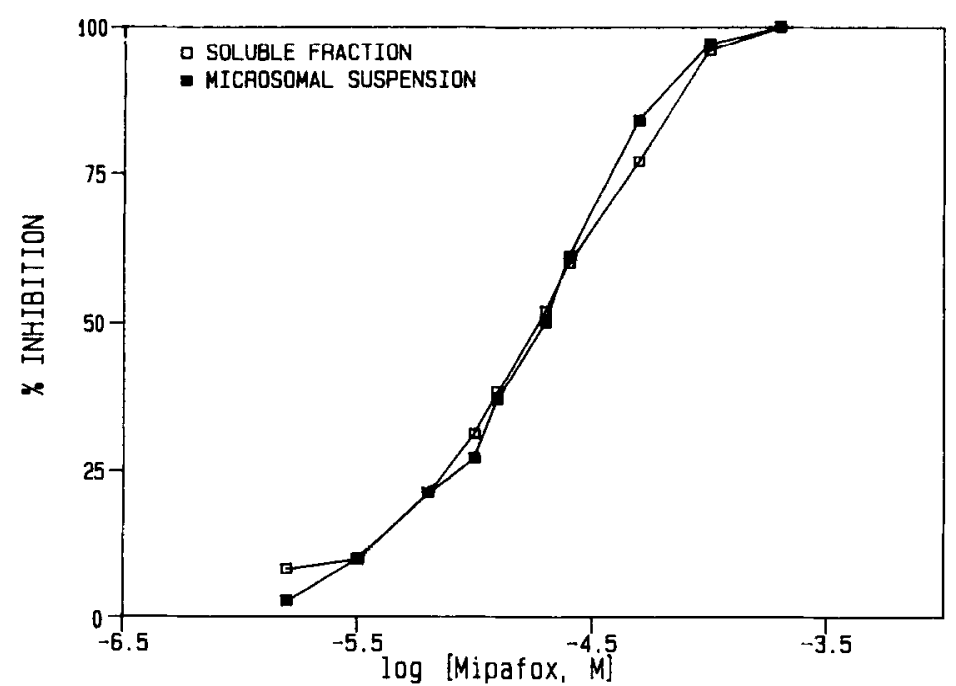

Pigure 2. Mipafox titration curves of NTE activity in a hen brain microsomal suspension in $40 \%$ DMSO ( $/ v$ in water) (closed squares) and NTE activity in the soluble fraction following centrifugation at $100,000 \times \mathrm{g}$ for $60 \mathrm{~min}$ at $4^{\circ} \mathrm{C}$ (open squares). The $\mathrm{PI}_{50}$ for each curve is 4.72. Data are duplicate determinations from a single experiment.

recentrifuged supernatants $(259 \pm 54 \mathrm{ug} / \mathrm{m} 1)$, are not statistically significant $(p=0.05 ; n=6)$.

\section{DISCUSSION}

Aqueous $40 \%$ DMSO was shown in this study to be capable of solubilizing enzymatically active NTE with retention of its native response to inhibitors: this is the first report of successful solubilization of active NTE by an organic solvent. Such a procedure provides some potential advantages over detergent-solubilized preparations for enzyme purification and membrane reconstitution. Because of the micellar nature of detergent systems, detergents require dialysis or gel permeation for their removal, whereas organic solvents may be simply evaporated in vacuo (13). Kinetic studies of NTE have also been hampered because neither the enzyme nor its lipophilic substrates are soluble in aqueous solution and must be suspended in detergent micelles (14). Thus, previous estimates of $\mathrm{Km}$ values for substrates have been based on nominal rather than true concentrations in solution (2).

The loss of NTE activity without significant loss of protein in the supernatant upon further centrifugation is an interesting observation that remains unexplained. Assays on all aliquots were performed at the same time, 
so the phenomenon appears to be due to centrifugation itself rather than time dependent loss of activity. Similar experiments have not been done with detergent-solubilized NTE, but it would be of interest to do so.

IRE activity was somewhat more soluble than NTE activity in $40 \%$ DMSO, but in view of the large amount of total protein solubilized, it is doubtful that attempts to optimize DMSO solubilization to take advantage of selective extraction would be fruitful.

Other studies have shown DMSO to be a powerful membrane disrupter (16), yet serine hydrolases have been shown to retain activity in this solvent (17). These observations are in accord with our results and suggest that DMSO extraction should be a useful adjunct to more traditional approaches to the solubilization of NTE.

\section{ACKNONLEDGEMENTS}

This work was supported, in part, by research grants 2 R01 ES01611 and 1 R01 ES02770 from the National Institutes of Health/ Nationa1 Institute of Environmental Health Sciences. The authors thank Dr. Frank Greco, Laboratory of Physical Chemistry, Department of Pathology, New England Deaconess Hospital, Boston, MA for extremely useful discussions of the data.

\section{REFBRENCES}

1. Davis, C.S. and Richardson, R.J. (1980) in Experimental and Clinical Neurotoxicology pp. 527-544 (Spencer, P.S. and Schaumburg, H.H., eds) Williams and Wilkins, Baltimore.

2. Johnson, M.K. (1982) Rev. Biochem. Toxico1. 4, 141-212.

3. Johnson, M.K. and Lauwerys, R. (1969) Nature 222, 1066-1067.

4. Johnson, M.K. (1974) J. Neurochem. 23, 785-789.

5. Clothier, B. and Johnson, M.K. (1979) Biochem. J. 177, 549-588.

6. Clothier, B. and Johnson, M.K. (1980) Biochem. J. 185, 739-747.

7. Williams, D.G. (1983) Biochemical J. 209, 817-829.

8. Richardson, R.J., Davis, C.S. and Johnson, M.K. (1979) J. Neurochem. 32, 607-615.

9. Johnson, M.K. (1971) Biochem. J. 122, 51P-52P.

10. Davis, C.S., Barth, M.L., Dudek, B.R., and Richardson, R.J. (1980) in Mechanisms of Toxicity and Hazard Evaluation pp. 63-67 (Holmstedt, B., Lauwerys, R., Mercier, M. and Roberfroid, M., eds) Elsevier, Amsterdam.

11. Ishikawa, Y., Chow, E., McNamee, M.G., McChesney, M., and Wilson, B.W. (1983) Toxicol. Let. 17, 315-320.

12. Chemnitius, J.M. and Zech, R. (1984) Int J. Biochem. 4, 361-367.

13. Klausner, R.D., van Renswoude, J., Blumentha1, R., and Rivnay, B. (1984) in Receptor Biochemistry and Methodology, Vol. 3, pp. 209-239 (Venter, J.C. and Harrison, L.C., eds) Alan R. Liss, Inc., New York.

14. Johnson, M.K. (1977) Arch. Toxicol. 37, 113-115.

15. Bradford, M.M. (1976) Analyt. Biochem. 72, 248-254.

16. Koh1, B. and Sanderman,H. (1977) FEBS Let. 80, 408-412.

17. Schwartz, H.M., Wu, W-S., Marr, P.W., and Jones, J.B. (1978) J. Am. Chem. Soc. $100,5199-5203$. 